\title{
Ye'elimite synthesis by chemical routes and role of iron
}

\author{
Fatima-Zahra Abir ${ }^{1,2,3^{*}}$, Mohamed Mesnaoui ${ }^{2,4}$, Younes Abouliatim ${ }^{3}$, Lhbib $_{\text {Nibou }}^{5}$, \\ Youssef El Hafiane ${ }^{1}$, and Agnès Smith ${ }^{1}$
}

${ }^{1}$ University of Limoges, Institute of Research for Ceramics (IRCER), UMR 7315, European Ceramics Center, 12 Avenue Atlantis, 87068, Limoges Cedex, France

${ }^{2}$ Laboratory of Materials Science and Process Optimization FSSM - University of Cadi Ayyad, Marrakech 40000, Morocco

${ }^{3}$ Laboratory of Materials, Processes, Environment and Quality, ENSA, University of Cadi Ayyad, Safi, Morocco.

${ }^{4}$ Center for Soil and Fertilizer Research in Africa (CESFRA) - Mohammed VI Polytechnic University, Benguerir, Morocco

${ }^{5}$ Systems \& Applications Engineering Laboratory (LISA) - University of Cadi Ayyad, ENSA, Marrakech, Morocco

\begin{abstract}
The cement industry has been taking significant steps for years to reduce its carbon footprint by opting for alternative less polluting materials such as sulfo-aluminous cements (CSA). These binders, compared to ordinary Portland cements (OPC), have two advantages: reduction of the $\mathrm{CO}_{2}$ emissions and energy saving because the sintering temperature of CSA cements is much lower than ordinary cement (Portland). The aim of this work is to study the effect of iron oxide on the formation of the ye'elimite phase, which represents the main phase of (CSA). This study details the protocol for the chemical synthesis of ye'elimite containing increasing amounts of iron (general formula: $\mathrm{Ca}_{4} \mathrm{Al}_{(6-2 x)} \mathrm{Fe}_{2 x} \mathrm{SO}_{16}$ with $\mathrm{x}=0.00$ to 1.13). The maximum ye'elimite content is reached at a sintering temperature of $1250{ }^{\circ} \mathrm{C}$. The presence of iron promotes the formation of cubic ye'elimite at the expense of the orthorhombic phase. The total incorporation of iron in ye'elimite structure is possible when $\mathrm{x}<0.12$. Beyond this content, the ferritic phase $(\mathrm{CaO})_{2}\left(\mathrm{Al}_{2} \mathrm{O}_{3}, \mathrm{Fe}_{2} \mathrm{O}_{3}\right)$ appears as a minor phase and its quantity becomes more important with the increase of the percentage of iron introduced in the synthesis. Finally, the electron microscopy allows to observe nanometric grains assembled in larger aggregates.
\end{abstract}

* Corresponding author: fatima-zahra.abir@unilim.fr 